\title{
Right ventricular reverse remodelling after balloon pulmonary angioplasty
}

\author{
Shigefumi Fukui', Takeshi Ogo'1, Yoshiaki Morita², Akihiro Tsuji ${ }^{1}$, Emi Tateishi², \\ Kumi Ozaki², Yoshihiro Sanda², Tetsuya Fukuda², Satoshi Yasuda¹, Hisao Ogawa \\ and Norifumi Nakanishi ${ }^{1}$
}

Affiliations: 'Dept of Cardiovascular Medicine, National Cerebral and Cardiovascular Center, Suita, and ${ }^{2}$ Dept of Radiology, National Cerebral and Cardiovascular Center, Suita, Japan.

Correspondence: T. Ogo, Dept of Cardiovascular Medicine, National Cerebral and Cardiovascular Center, 7-1 Fujishirodai 5-chome, Suita 565-8565, Japan. E-mail: ogo.takeshi.hpamail.ncvc.go.jp

ABSTRACT Balloon pulmonary angioplasty (BPA) has been reported to improve haemodynamics and functional capacity, with an acceptable risk, in patients with chronic thromboembolic pulmonary hypertension (CTEPH) who are not candidates for pulmonary endarterectomy. However, right ventricular (RV) function, an important predictor in CTEPH, remains to be elucidated. We aimed to examine the impact of BPA on RV remodelling and dysfunction relative to haemodynamic improvements in patients with inoperable CTEPH.

20 consecutive patients with inoperable CTEPH who underwent BPA with cardiovascular magnetic resonance before and after BPA were retrospectively studied.

BPA led to significant amelioration of the mean pulmonary arterial pressure, cardiac index and pulmonary vascular resistance (PVR), without death or major complications. Furthermore, BPA significantly ameliorated right-sided heart failure symptoms and signs, and exercise capacity. Cardiovascular magnetic resonance revealed a marked improvement in RV end-diastolic and end-systolic volume index, with concomitant improvements in RV ejection fraction, mass and interventricular septal bowing after BPA. Changes in RV volumes strongly correlated with changes in cardiac index and PVR.

BPA induced RV reverse remodelling and improved systolic dysfunction safely by ameliorating haemodynamics in patients with inoperable CTEPH. Evaluating RV function with cardiovascular magnetic resonance may be effective for noninvasively monitoring BPA efficacy.

@ERSpublications

BPA safely ameliorates haemodynamics, leading to right ventricular reverse remodelling in inoperable CTEPH http://ow.ly/tQX2R

For editorial comments see page 1230 .

This article has supplementary material available from www.erj.ersjournals.com

Received: Jan 172014 | Accepted after revision: Feb 122014 | First published online: March 132014

Conflict of interest: None declared.

Copyright @ERS 2014 


\section{Introduction}

Chronic thromboembolic pulmonary hypertension (CTEPH) is a life-threatening condition that leads to progressive right-sided heart failure and a poor prognosis if left untreated [1-3]. Pulmonary endarterectomy (PEA) is an established treatment for CTEPH with an acceptable mortality $[4,5]$. However, a limited post-operative reduction in pulmonary vascular resistance (PVR) occurs in some patients with distal, surgically inaccessible thrombi or significant small vessel arteriopathy $[1,6]$. Recently, balloon pulmonary angioplasty (BPA) has been reported to significantly decrease mean pulmonary arterial pressure (mPAP) and PVR with an acceptable procedure-related risk and, subsequently, improve the functional status and exercise capacity in patients who are not candidates for PEA due to distal-type CTEPH, severe concomitant comorbidity, or residual pulmonary hypertension after PEA [7-10].

Right ventricular (RV) function has a significant impact on the prognosis of CTEPH and other forms of pulmonary arterial hypertension (PAH) [11]. Preoperative RV dysfunction in CTEPH patients is also reported to be associated with increased post-operative mortality after PEA $[4,12,13]$. However, whether or not BPA ameliorates advanced RV remodelling and dysfunction resulting from long-standing pressure overload in CTEPH patients remains unknown. Cardiovascular magnetic resonance (CMR) is considered the standard modality for noninvasive and reproducible assessment of RV volume and function; other methods, such as echocardiography and computed tomography (CT), seem to be of low value in accurately evaluating the complex structure and functional impairment of the right ventricle [14-16].

Thus, the aim of the present study was to determine the impact of BPA on advanced RV remodelling and dysfunction, relative to haemodynamic improvements, using CMR in patients with inoperable CTEPH.

\section{Methods}

\section{Study subjects}

We retrospectively studied 20 consecutive patients (mean \pm SD (range) age $67 \pm 9$ (44-82) years; including 15 females) with inoperable CTEPH who underwent BPA and whose quantitative recordings of CMR were obtained between August 2012 and December 2013. All patients complained of dyspnoea on effort that was more severe than World Health Organization functional class (WHO-FC) II. Patients who could not remain in a supine position for $>20 \mathrm{~min}$ because of dyspnoea were excluded in the present study as they could not undergo CMR. Patients were diagnosed with CTEPH using the standard criteria as previously described $[17,18]$. They were considered to have inoperable disease if they had distal, surgically inaccessible thrombi or severe concomitant medical comorbidity based on a detailed medical history, physical examination, ECG, chest radiograph, echocardiograph, lung ventilation/perfusion scintigraphy, CT scan, CMR imaging, right heart catheterisation (RHC), and pulmonary digital subtraction angiography. Selection of BPA versus PEA was confirmed by the CTEPH team at our centre (National Cerebral and Cardiovascular Center, Suita, Japan), including radiologists and surgeons with expertise in performing PEA [19]. Adequate anticoagulation therapy was maintained for at least 6 months before BPA and was continued thereafter. RHC and CMR were performed before the first BPA session and at a mean \pm SD (range) of $4.0 \pm 0.8$ (3-6) months after the final BPA session. A clinical assessment, including functional status, brain natriuretic peptide (BNP) level, and 6-min walk distance (6MWD), was also performed simultaneously with the aforementioned procedures. Written informed consent was obtained from all patients, and the institution's ethics committee also approved the present study.

\section{BPA procedure}

BPA was performed via the internal jugular vein to treat branches in the right pulmonary artery or via the femoral vein to treat branches in the left pulmonary artery, considering the technical ease of steering the wires and catheters. The targeted vessel was selected on the basis of several characteristic findings, such as webs, bands, abrupt narrowing and complete obstructions detected by other modalities, including CT and pulmonary digital subtraction angiography, in addition to accessibility. We selectively introduced a 6-French multipurpose guiding catheter (Mach1 Peripheral MP; Boston Scientific, Natick, MA, USA) in a 6-French long sheath (BRITE TIP sheath introducer; Cordis, New Brunswick, NJ, USA) with a soft-tipped 0.035 -inch wire into the targeted vessel. After crossing a 0.014-inch guide wire (Cruise; Asahi Intecc, Tokyo, Japan) through the targeted lesion, we carefully inflated the balloons (IKAZUCHI PAD; Kaneka, Osaka, Japan) until the indentation disappeared. The appropriate size of a balloon (range 2.0-7.0 mm) was determined based upon a targeted vessel diameter measured by CT, in addition to angiographic findings. The treatment during the first session was limited to one to two segments in one lobe to prevent severe reperfusion pulmonary oedema after BPA. Upon BPA completion, we carefully observed patient condition in terms of symptoms, vital signs, oxygenation and haemodynamics with a 9-French Swan-Ganz catheter (Swan-Ganz CCO CEDV; Edwards Lifesciences, Irvine, CA, USA) overnight in the intensive care unit. We routinely used nonivasive continuous positive airway pressure ventilation after BPA overnight. Additional 
BPA sessions were recommended for each patient until a mPAP of $<30 \mathrm{mmHg}$ was achieved and each BPA session was repeated at a mean (range) interval of $5.2 \pm 6.7$ (1-20) weeks.

\section{Haemodynamic studies}

RHC, together with pulmonary digital subtraction angiography, was performed according to standard procedures in order to measure mean pulmonary capillary wedge pressure, mPAP, mean right atrial pressure (RAP), cardiac output, PVR and total pulmonary resistance (TPR) as previously described [17]. Cardiac output was determined by the Fick method and corrected for body surface area.

\section{CMR image acquisition and analysis}

CMR imaging was performed with a standardised clinical protocol on a 1.5-T system (Magnetom Sonata; Siemens, Erlangen, Germany). We acquired the cine imaging using a true-FISP sequence (echo time $1.3 \mathrm{~ms}$, repetitive time $2.6 \mathrm{~ms}$, flip angle $60^{\circ}$, slice thickness $8 \mathrm{~mm}$, gaps $2 \mathrm{~mm}$, in plane resolution $4.17 \times 2.73 \mathrm{~mm}$ ) with multiple breath-holds in contiguous short-axis and transaxial slices that encompassed both ventricles, and three standard long-axis slices. Prospective ECG gating was performed using the R-wave as a trigger.

To quantify the RV end-diastolic volume, RV end-systolic volume, RV stroke volume, RV ejection fraction (RVEF) and mass, two experienced radiologists manually traced the RV endocardial and epicardial contours in the end-systolic and end-diastolic frames of transaxial slices using dedicated software (Argus; Siemens). Left ventricular (LV) volumes were determined in the same manner, but using short-axis slices. The ventricular mass index (VMI) was defined as the RV mass divided by the LV mass [20]. The degree of interventricular septal bowing was expressed as a ratio of the RV diameter to biventricular diameter in the early diastolic phase of the ventricle using short-axis slices, as previously described [21]. Cardiac volumes and mass were all corrected for body surface area.

\section{Statistical analysis}

Quantitative results are expressed as mean \pm SD. Changes in haemodynamics, CMR and other clinical parameters were compared using a paired t-test or Wilcoxon signed-ranks test, as appropriate. The correlations between variables were assessed by linear regression analysis. A p-value $<0.05$ was considered statistically significant. All statistical analyses were performed with JMP 10.0 (SAS Institute, Cary, NC, USA).

\section{Results}

\section{Baseline patient characteristics}

For this study, we enrolled 20 consecutive patients (mean \pm SD (range) age $67 \pm 9$ (44-82) years; including 15 females), with a mean WHO-FC score of 2.8 (table 1). Only one patient had prior history of PEA (table 1), whereas the remaining 19 patients were considered inoperable due to: surgically inaccessible thrombi $(n=16)$, severe concomitant medical comorbidity $(n=2)$, or refusal of PEA $(n=1)$. The mean duration from onset of symptoms (disease duration) to BPA was mean \pm SD $60 \pm 63$ (range 6-276) months (table 1). Before BPA, 15 (75\%) patients had been treated with oral pulmonary hypertension-specific therapies consisting of a endothelin receptor antagonist $(n=6)$, an oral prostacyclin analogue $(n=13)$, a phosphodiesterase type-5 inhibitor $(n=4)$, and oral combination therapy $(n=6)$ (table 1). Despite comprehensive medical treatment, including home oxygen therapy, anticoagulation therapy and therapy with diuretics, mPAP and PVR at baseline were elevated to $39.4 \pm 7.6 \mathrm{mmHg}$ and $889 \pm 365 \mathrm{dyn} \cdot \mathrm{s}^{-1} \cdot \mathrm{cm}^{-5}$, respectively, along with a reduced cardiac index $\left(2.18 \pm 0.65 \mathrm{~L} \cdot \mathrm{min}^{-1} \cdot \mathrm{m}^{-2}\right)($ table 2$)$. However, RAP, one of the important prognostic predictors in pulmonary hypertension $[22,23]$, was within normal range (table 2). The disease duration before BPA positively correlated with baseline mPAP (fig. S1a), but did not correlate with any other haemodynamic or RV functional parameters derived from RHC or CMR.

\section{BPA and haemodynamic parameters}

BPA consisted of a mean \pm SD (range) of $3.2 \pm 0.9$ (2-5) procedures for each patient. All procedures were successfully performed without any deaths or major complications, such as severe reperfusion pulmonary oedema requiring invasive mechanical ventilation, during the follow-up (fig. 1). The mean PAP, PVR and TPR levels were markedly ameliorated by BPA, with concomitant improvements in the cardiac index and mixed venous oxygen saturation (table 2). RAP was within the normal range at baseline and significantly decreased after BPA (table 2).

\section{Clinical assessment}

After BPA, the average WHO-FC and BNP level significantly decreased and the 6MWD significantly increased (table 2). 
TABLE 1 Baseline characteristics of enrolled patients with inoperable chronic thromboembolic pulmonary hypertension

Baseline

$\begin{array}{lc}\text { Subjects } & 20 \\ \text { Age years } & 67 \pm 9(44-82) \\ \text { Female/male } & 15 / 5 \\ \text { WHO-FC I/II/III/IV } & 0 / 6 / 13 / 1 \\ \text { Patients previously treated with PEA } & 1 \\ \text { Duration from onset of symptoms before BPA months } & 60 \pm 63(6-276) \\ \text { Previous PH-specific therapy" } & 15(75) \\ \text { Endothelin receptor antagonist } & 6 \\ \text { Oral prostacyclin analogue } & 13 \\ \text { Phosphodiesterase type-5 inhibitor } & 4 \\ \text { Oral combination therapy } & 6\end{array}$

Data are presented as $\mathrm{n}$, mean \pm SD (range) or $\mathrm{n}(\%)$. WHO-FC: World Health Organization functional class; PEA: pulmonary endarterectomy; BPA: balloon pulmonary angioplasty; PH: pulmonary hypertension. \#: some patients received combination $\mathrm{PH}$-specific therapy, thus, the data for each therapy do not total 15 .

Right ventricular function

At baseline, CMR revealed prominent increases in the RV end-diastolic volume index (RVEDVI) and RV end-systolic volume index (RVESVI), with concomitant reductions in the RVEF (fig. 2, table 3). RV systolic dysfunction, defined as an RVEF $<45 \%$, was observed in 17 (85\%) patients before BPA, whereas no LV systolic dysfunction was present. Both the RVEDVI and RVESVI were significantly reduced after BPA, with concomitant improvements in the RVEF (fig. 2, table 3). Moreover, the RV mass index (RVMI), VMI and septal inversion ratio also significantly improved after BPA (fig. 2, table 3). There were no significant changes in LV volumes or function (table 3). Changes in the RV volumes (RVEDVI and RVESVI) were strongly correlated with those for cardiac index and pulmonary vascular resistance (PVR and TPR) (figs 3 and S2a and b) and were also significantly correlated with those in the RV preload (RAP) (fig. S2c and d). There were no significant correlations between the disease duration before BPA and changes in any of the parameters reflecting RV volumes, function or haemodynamics (fig. S1b-d).

TABLE 2 Clinical and haemodynamic data before and after balloon pulmonary angioplasty (BPA)

\begin{tabular}{|c|c|c|c|}
\hline & Before BPA & After BPA & p-value \\
\hline Subjects & 20 & 20 & \\
\hline WHO-FC & 2.8 & 2.0 & $<0.001$ \\
\hline \multicolumn{4}{|l|}{ Haemodynamic data } \\
\hline Mean PCWP $\mathrm{mmHg}$ & $6.5 \pm 2.5$ & $6.4 \pm 2.6$ & 0.82 \\
\hline Mean PAP mmHg & $39.4 \pm 7.6$ & $27.3 \pm 8.5$ & $<0.001$ \\
\hline Mean RAP $\mathrm{mmHg}$ & $3.7 \pm 2.2$ & $2.5 \pm 2.4$ & $<0.05$ \\
\hline Cardiac index $\mathrm{L} \cdot \mathrm{min}^{-1} \cdot \mathrm{m}^{-2}$ & $2.18 \pm 0.65$ & $2.38 \pm 0.46$ & $<0.05$ \\
\hline PVR dyn $\cdot \mathrm{s}^{-1} \cdot \mathrm{cm}^{-5}$ & $889 \pm 365$ & $490 \pm 201$ & $<0.001$ \\
\hline $\mathrm{TPR}$ dyn $\cdot \mathrm{s}^{-1} \cdot \mathrm{cm}^{-5}$ & $1066 \pm 441$ & $639 \pm 237$ & $<0.001$ \\
\hline Heart rate beats $\cdot \min ^{-1}$ & $75 \pm 13$ & $65 \pm 10$ & $<0.001$ \\
\hline $\mathrm{SVO}_{2} \%$ & $61.9 \pm 8.3$ & $65.8 \pm 5.4$ & $<0.05$ \\
\hline 6MWD m & $361+104^{\#}$ & $463 \pm 76^{\#}$ & $<0.001$ \\
\hline BNP level $\mathrm{pg} \cdot \mathrm{mL}^{-1}$ & $175 \pm 135$ & $56 \pm 49$ & $<0.001$ \\
\hline
\end{tabular}

Data are presented as $n$, mean or mean \pm SD, unless otherwise stated. WHO-FC: World Health Organization functional class; PCWP: pulmonary capillary wedge pressure; PAP: pulmonary arterial pressure; RAP: right atrial pressure; PVR: pulmonary vascular resistance; TPR: total pulmonary resistance; $\mathrm{SVO}_{2}$ : mixed venous oxygen saturation; 6MWD: 6-min walk distance; BNP: brain natriuretic peptide. ${ }^{\#}: \mathrm{n}=17$ as three patients could not perform the 6MWD due to dyspnoea. 

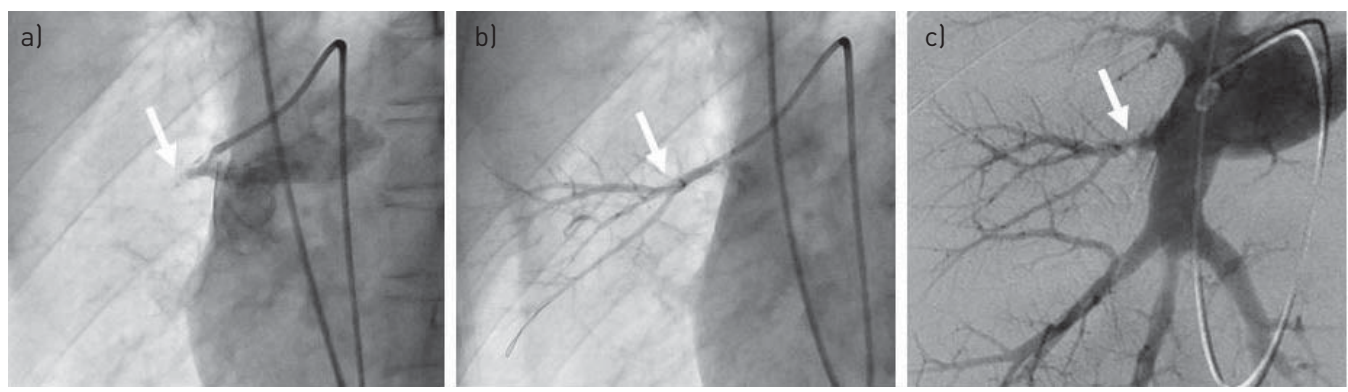

FIGURE 1 Balloon pulmonary angioplasty in distal-type chronic thromboembolic pulmonary hypertension (CTEPH). Representative angiography a) before, b) immediately after and c) at follow-up for a characteristic lesion with complete obstruction in a patient with inoperable CTEPH. There was no evidence of restenosis in the treated lesion (arrow) at follow-up.

\section{Discussion}

The novel findings of the present study are that: 1) BPA ameliorates advanced RV remodelling and dysfunction caused by long-standing pressure overload in CTEPH patients who are not surgical candidates; and 2) the improvements in RV remodelling were strongly correlated with those in haemodynamic parameters. To the best of our knowledge, this is the first study that demonstrates the impact of BPA on advanced RV remodelling and dysfunction relative to haemodynamic parameter improvements in patients with inoperable CTEPH.

\section{Beneficial effects of BPA on haemodynamics and clinical status}

The first report describing the use of BPA in a series of patients with inoperable CTEPH demonstrated that BPA significantly reduced PAP, but was associated with a high rate of lethal complications, including severe reperfusion pulmonary oedema [7]. Thereafter, the use of BPA was not as widespread as expected due to concerns about procedure-related complications. However, several studies have recently demonstrated the technical evolution of the procedure, maintaining its efficacy while improving its safety [8-10, 24, 25]. Briefly, Mizoguchi et al. [8] reported a refined, low-mortality BPA procedure that improved the clinical status and haemodynamics of patients with inoperable CTEPH. SUGIMURA et al. [9] demonstrated the usefulness of optical coherence tomography in percutaneous transluminal pulmonary angioplasty. In the present study, BPA significantly and sufficiently improved mPAP and PVR with concomitant improvements in the cardiac index in the selected patients with inoperable CTEPH, without any deaths or major complications. Moreover, BPA significantly ameliorated right-sided heart failure symptoms and signs, and improved exercise capacity, all of which are important indicators of the clinical status of CTEPH patients, as evidenced by improved WHO-FC, BNP level and 6MWD. In addition, BPA also significantly improved RAP, which was within normal limits even at baseline in the present study. This finding might reflect the fact that volume overload and right-sided heart failure had been stabilised with medical treatment, including diuretics, before BPA and that, as mentioned previously, more severe patients with elevated RAP were excluded from the present study because they could not maintain a supine position during CMR. Taken together, BPA is a safe and effective therapy for selected patients with inoperable CTEPH to ameliorate patient haemodynamics and clinical status.

\section{Beneficial effects of BPA on RV remodelling and function}

One of the major prognostic determinants in patients with pulmonary hypertension is RV dysfunction $[11,26,27]$. Long-term pressure overload in CTEPH patients induces progressive RV remodelling, including myocardial hypertrophy, ventricular dilatation, tricuspid regurgitation and early diastolic ventricular septal bowing. These morphological changes result in RV dysfunction and subsequent failure, which are the most common causes of death in patients with CTEPH and other forms of PAH [26]. REESINK et al. [28] described RV reverse remodelling after hemodynamically successful PEA in CTEPH patients using CMR. In addition, IINO et al. [21] reported a time course of the restoration for RV volumes and ejection fraction of up to 6 months after PEA. However, there is a lack of data on the efficacy of BPA for RV remodelling and dysfunction in CTEPH patients who are not surgical candidates because of the location of thrombi or serious concomitant comorbidity. In the present study, baseline CMR revealed prominent RV remodelling and systolic dysfunction, as evidenced by obvious increases in RVEDVI and RVESVI with concomitant RVEF reduction. Nevertheless, BPA significantly ameliorated RV dilatation (RVEDVI and RVESVI), RV systolic dysfunction (RVEF), RV hypertrophy (RVMI and VMI), and interventricular septal bowing (septal inversion ratio). Furthermore, there were strong correlations between changes in RV 
End diastole


End systole
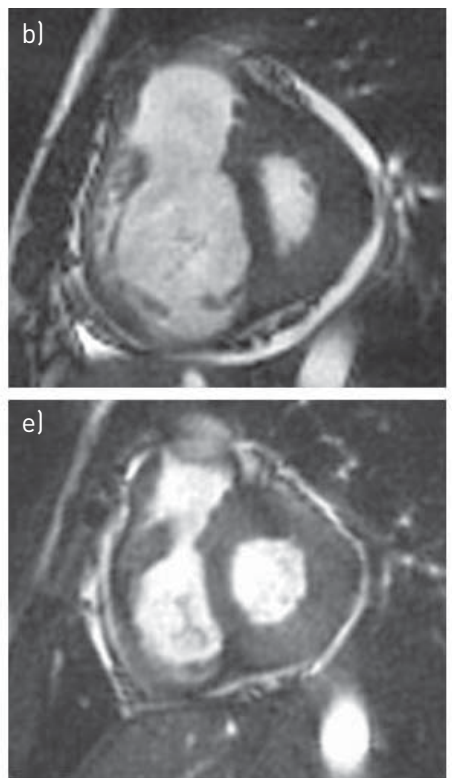

Early diastole
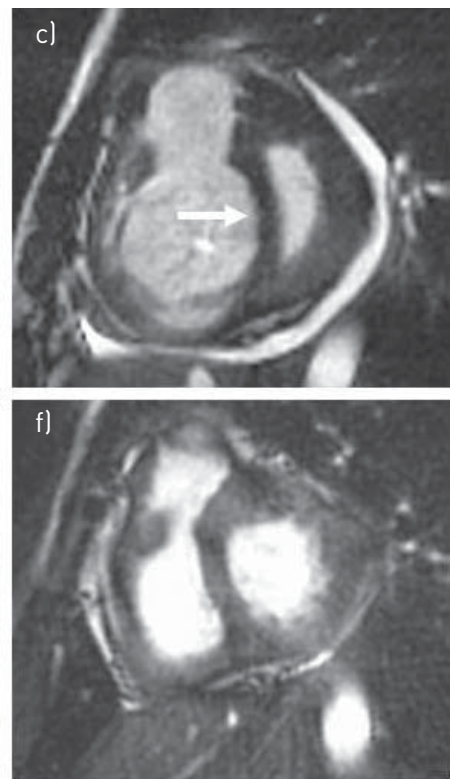

FIGURE 2 Balloon pulmonary angioplasty (BPA) ameliorates right ventricular (RV) dilatation, hypertrophy and leftward septal inversion. Representative cine images of cardiovascular magnetic resonance obtained from short-axis slices in a patient with inoperable chronic thromboembolic pulmonary hypertension at a, d) end diastole, b, e) end systole and c, f) early diastole of the ventricle before $(\mathrm{a}-\mathrm{c})$ and after $(\mathrm{d}-\mathrm{f})$ BPA. There is prominent RV dilatation, hypertrophy and interventricular septal bowing (arrow) before BPA, all of which improved after BPA.

remodelling, measured with CMR, and changes in most haemodynamic parameters derived from RHC. Together, these evidences indicate that BPA induces RV reverse remodelling and improves systolic dysfunction in relation to haemodynamic improvements in patients with inoperable CTEPH. Furthermore, the evaluation of RV function by CMR could predict the efficacy and outcome of BPA noninvasively, reproducibly and accurately.

TABLE 3 Cardiac volumes, function and mass before and after balloon pulmonary angioplasty (BPA)

Before BPA

Subjects

Right ventricle

RVEDVI $\mathrm{mL} \cdot \mathrm{m}^{-2}$

RVESVI $\mathrm{mL} \cdot \mathrm{m}^{-2}$

RVSVI $\mathrm{mL} \cdot \mathrm{m}^{-2}$

RVEF \%

$\mathrm{RV}$ mass index $\mathrm{g} \cdot \mathrm{m}^{-2}$

VMI ratio"

Septal inversion ratio

\section{Left ventricle}

LVEDVI $\mathrm{mL} \cdot \mathrm{m}^{-2}$

LVESVI $\mathrm{mL} \cdot \mathrm{m}^{-2}$

LVSVI $\mathrm{mL} \cdot \mathrm{m}^{-2}$

LVEF \%

$\mathrm{LV}$ mass index $\mathrm{g} \cdot \mathrm{m}^{-2}$

\section{0}

$$
\begin{gathered}
130 \pm 52 \\
89 \pm 43 \\
41 \pm 14 \\
34 \pm 9 \\
38 \pm 20 \\
0.79 \pm 0.33 \\
0.63 \pm 0.07
\end{gathered}
$$

$65 \pm 15$

$29 \pm 8$

$37 \pm 9$

$56 \pm 5$

$47 \pm 12$
After BPA

20

$92 \pm 24$
$55 \pm 18$
$37 \pm 10$
$41 \pm 8$
$29 \pm 12$
$0.62 \pm 0.20$
$0.58 \pm 0.05$

$69 \pm 12$

$32 \pm 8$

$38 \pm 7$

$55 \pm 6$

$45 \pm 10$ p-value

Data are presented as $n$ or mean $\pm S D$, unless otherwise stated. RVEDVI: right ventricular (RV) end-diastolic volume index; RVESVI: RV end-systolic volume index; RVSVI: RV stroke volume index; RVEF: RV ejection fraction; VMI: ventricular mass index; LVEDVI: left ventricular (LV) end-diastolic volume index; LVESVI: LV endsystolic volume index; LVSVI: LV stroke volume index; LVEF: LV ejection fraction. " : defined as RV mass divided by LV mass; ": ratio of RV diameter to biventricular diameter in early diastolic phase of the ventricle. 

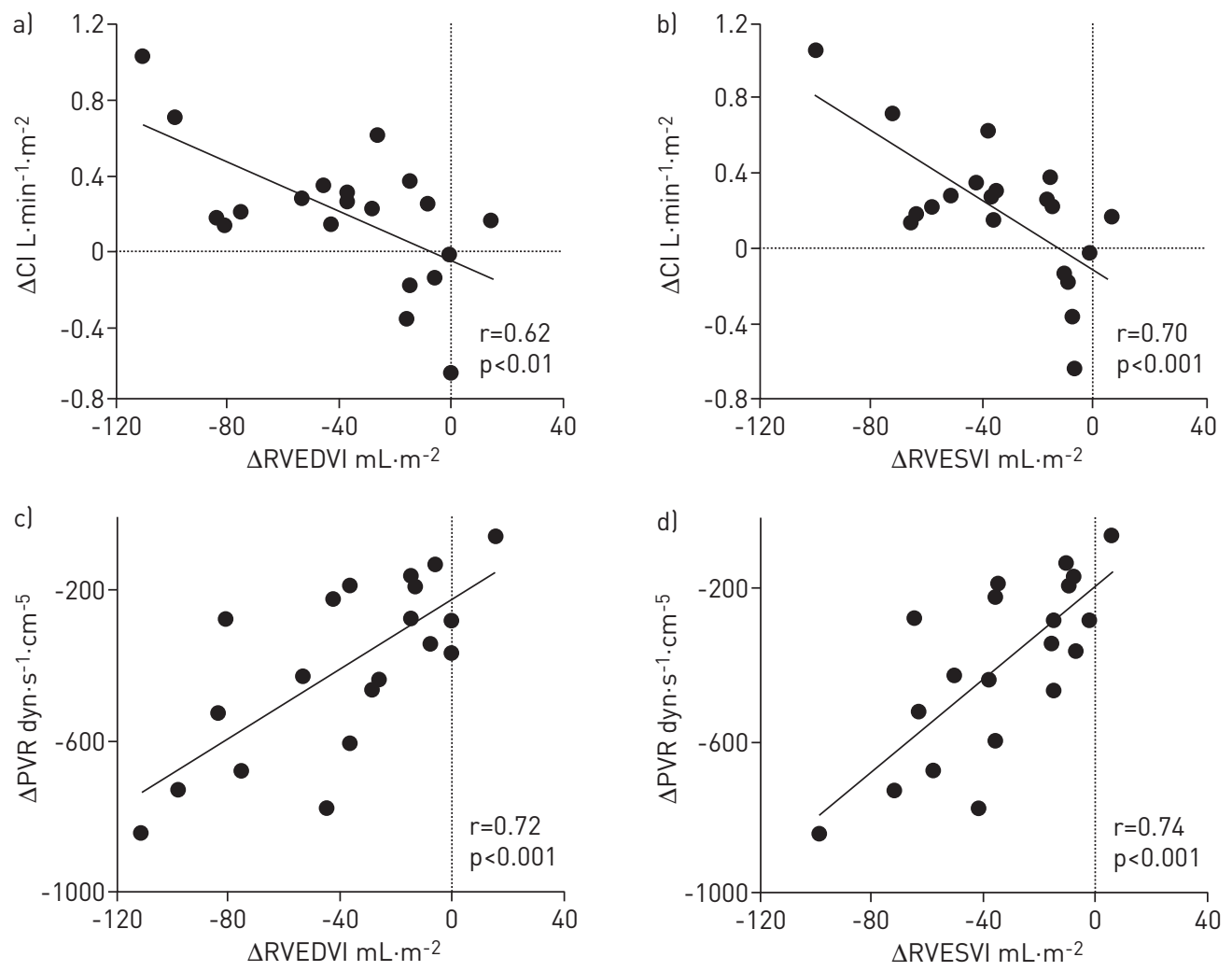

FIGURE 3 The correlation of right ventricular (RV) volumes against cardiac output and pulmonary vascular resistance (PVR). The changes in a, c) RV end-diastolic volume index (RVEDVI) and b, d) RV end-systolic volume index (RVESVI), measured noninvasively via cardiovascular magnetic resonance, strongly correlated with changes in $\mathrm{a}, \mathrm{b}$ ) cardiac index (CI) and c, d) PVR obtained invasively from right heart catheterisation in 20 patients with inoperable chronic thromboembolic pulmonary hypertension.

However, this study found that BPA did not significantly affect LV volumes and function, which is consistent with the findings on PEA of IINO et al. [21] and KREITNER et al. [16], but inconsistent with those of REESINK et al. [28] and HARDZIYENKA et al. [29]. Some pulmonary hypertension reports have demonstrated that LV systolic and diastolic dysfunction may occur secondary to RV dysfunction through ventricular interaction, ventricular interdependence, early diastolic ventricular septal bowing, and hypertrophy of the interventricular septum [28, 30]. vAN WOLFEREN et al. [27] reported that LV enddiastolic volume index significantly increased from 41 to $46 \mathrm{~mL} \cdot \mathrm{m}^{-2}$ after 1 year of medical treatment, including intravenous epoprostenol or endothelin receptor antagonists, in patients with idiopathic PAH, and reduced LV volume is a strong predictor of poor survival and treatment failure. Their patients had higher mPAP (56 versus $39 \mathrm{mmHg}$ ) and smaller $\mathrm{LV}\left(41\right.$ versus $65 \mathrm{~mL} \cdot \mathrm{m}^{-2}$ ) at baseline, perhaps reflecting more severe compression of the LV, as compared with the present study [27]. Thus, whether or not LV enddiastolic volume index significantly increases after treatment despite comparable change in LV end-diastolic volume index (5 versus $4 \mathrm{~mL} \cdot \mathrm{m}^{-2}$ ) might depend, in part, on the severity of baseline haemodynamics; although it is necessary to take into account that the underlying diseases and treatments are different between two studies. Future, larger studies will be needed to determine whether this discrepancy with previous reports can also be attributed to the differences between BPA and PEA in the measurement of cardiac function with CMR between institutions, the number and backgrounds of the enrolled patients, or other reasons.

The patients enrolled in the present study had longer average disease duration (60 months) than patients in previous studies (28-43 months) where the efficacy of PEA for RV function or BPA for haemodynamics has been examined $[7-9,21,29,31]$. This indicates that the patients in the present study had suffered from RV pressure overload for a long time and were only given medical therapy; consequently, the present patients might have had advanced RV remodelling. The disease duration before BPA was positively correlated with the baseline mPAP, but not with any other haemodynamic or RV functional parameters, implying the increase of organised thromboembolic material itself or the existence of prevalent small vessel arteriopathy was a consequence of unobstructed area over-perfusion in patients with long durations of disease. The fact 
that the disease duration did not correlate with any baseline RV function parameters measured with CMR may suggest the variability of time-course in RV adaptation among patients as previously reported [3, 32]. However, the disease duration before BPA was not significantly correlated with changes in any parameters related to RV volumes, function or haemodynamics. These results suggest that BPA efficacy might be independent of the disease duration or stage.

\section{Limitations of the study}

Several limitations of the present study should be mentioned. First, this study was performed retrospectively, without any control subjects. Moreover, the number of patients enrolled in the study was small because we required CMR recordings both before and after a series of BPA procedures to permit an evaluation of RV function and its changes. Therefore, these results need to be confirmed in a larger, prospective study that includes comparison with patients who underwent PEA. Secondly, in the present study, we followed patients for a mean of 4 months to focus on RV function and its changes before and after BPA. Consequently, we did not address the long-term prognoses after BPA. In addition, we could not conclude whether or not the BPA-induced amelioration of haemodynamics and RV remodelling and function would be sustained or continue to improve gradually beyond 1 year. Therefore, the long-term survival, outcomes, efficacy and safety of BPA need to be addressed in a subsequent study. Finally, we did not assess myocardial changes in the RV, including fibrosis with delayed contrast enhancement, which might affect, in part, the RV function in CTEPH. Therefore, future studies need to clarify the detailed mechanisms whereby BPA ameliorates RV dysfunction.

\section{Conclusions}

The present study shows that BPA could be a safe and effective therapy for restoring RV remodelling and systolic dysfunction by ameliorating haemodynamic abnormalities in CTEPH patients who are not surgical candidates. This RV reverse remodelling induced by BPA was confirmed in patients with advanced RV dysfunction due to prolonged exposure to pressure overload. CMR is a useful modality for evaluating and following RV remodelling and function in CTEPH patients treated with BPA.

\section{References}

Piazza G, Goldhaber SZ. Chronic thromboembolic pulmonary hypertension. N Engl J Med 2011; 364: 351-360. Riedel M, Stanek V, Widimsky J, et al. Longterm follow-up of patients with pulmonary thromboembolism. Late prognosis and evolution of hemodynamic and respiratory data. Chest 1982; 81: 151-158.

3 Hoeper MM, Mayer E, Simonneau G, et al. Chronic thromboembolic pulmonary hypertension. Circulation 2006; 113: 2011-2020.

4 Jamieson SW, Kapelanski DP, Sakakibara N, et al. Pulmonary endarterectomy: experience and lessons learned in 1,500 cases. Ann Thorac Surg 2003; 76: 1457-1462.

5 Archibald CJ, Auger WR, Fedullo PF, et al. Long-term outcome after pulmonary thromboendarterectomy. Am J Respir Crit Care Med 1999; 160: 523-528.

6 Thistlethwaite PA, Madani M, Jamieson SW. Outcomes of pulmonary endarterectomy surgery. Semin Thorac Cardiovasc Surg 2006; 18: 257-264.

7 Feinstein JA, Goldhaber SZ, Lock JE, et al. Balloon pulmonary angioplasty for treatment of chronic thromboembolic pulmonary hypertension. Circulation 2001; 103: 10-13.

8 Mizoguchi H, Ogawa A, Munemasa M, et al. Refined balloon pulmonary angioplasty for inoperable patients with chronic thromboembolic pulmonary hypertension. Circ Cardiovasc Interv 2012; 5: 748-755.

9 Sugimura K, Fukumoto Y, Satoh K, et al. Percutaneous transluminal pulmonary angioplasty markedly improves pulmonary hemodynamics and long-term prognosis in patients with chronic thromboembolic pulmonary hypertension. Circ J 2012; 76: 485-488.

10 Kataoka M, Inami T, Hayashida K, et al. Percutaneous transluminal pulmonary angioplasty for the treatment of chronic thromboembolic pulmonary hypertension. Circ Cardiovasc Interv 2012; 5: 756-762.

11 Fedullo PF, Auger WR, Kerr KM, et al. Chronic thromboembolic pulmonary hypertension. Semin Respir Crit Care Med 2003; 24: 273-286.

12 Moser KM, Auger WR, Fedullo PF. Chronic major-vessel thromboembolic pulmonary hypertension. Circulation 1990; 81: 1735-1743.

13 Dartevelle P, Fadel E, Mussot S, et al. Chronic thromboembolic pulmonary hypertension. Eur Respir J 2004; 23: 637-648.

14 Vonk-Noordegraaf A, Souza R. Cardiac magnetic resonance imaging: what can it add to our knowledge of the right ventricle in pulmonary arterial hypertension? Am J Cardiol 2012; 110: Suppl. 6, 25S-31S.

15 Grothues F, Moon JC, Bellenger NG, et al. Interstudy reproducibility of right ventricular volumes, function, and mass with cardiovascular magnetic resonance. Am Heart J 2004; 147: 218-223.

16 Kreitner KF, Ley S, Kauczor HU, et al. Chronic thromboembolic pulmonary hypertension: pre- and postoperative assessment with breath-hold MR imaging techniques. Radiology 2004; 232: 535-543.

17 Iwase T, Nagaya N, Ando M, et al. Acute and chronic effects of surgical thromboendarterectomy on exercise capacity and ventilatory efficiency in patients with chronic thromboembolic pulmonary hypertension. Heart 2001; 86: $188-192$

18 Maeba H, Nakatani S, Sugawara M, et al. Different time course of changes in tricuspid regurgitant pressure gradient and pulmonary artery flow acceleration after pulmonary thromboendarterectomy: implications for discordant recovery of pulmonary artery pressure and compliance. Circ J 2007; 71: 1771-1775. 
19 Ogino $\mathrm{H}$, Ando M, Matsuda $\mathrm{H}$, et al. Japanese single-center experience of surgery for chronic thromboembolic pulmonary hypertension. Ann Thorac Surg 2006; 82: 630-636.

20 Saba TS, Foster J, Cockburn M, et al. Ventricular mass index using magnetic resonance imaging accurately estimates pulmonary artery pressure. Eur Respir J 2002; 20: 1519-1524.

21 Iino M, Dymarkowski S, Chaothawee L, et al. Time course of reversed cardiac remodeling after pulmonary endarterectomy in patients with chronic pulmonary thromboembolism. Eur Radiol 2008; 18: 792-799.

22 Thenappan T, Shah SJ, Rich S, et al. Survival in pulmonary arterial hypertension: a reappraisal of the NIH risk stratification equation. Eur Respir J 2010; 35: 1079-1087.

23 Benza RL, Miller DP, Gomberg-Maitland M, et al. Predicting survival in pulmonary arterial hypertension: insights from the Registry to Evaluate Early and Long-Term Pulmonary Arterial Hypertension Disease Management (REVEAL). Circulation 2010; 122: 164-172.

24 Inami T, Kataoka M, Shimura N, et al. Pulmonary edema predictive scoring index (PEPSI), a new index to predict risk of reperfusion pulmonary edema and improvement of hemodynamics in percutaneous transluminal pulmonary angioplasty. JACC Cardiovasc Interv 2013; 6: 725-736.

25 Andreassen AK, Ragnarsson A, Gude E, et al. Balloon pulmonary angioplasty in patients with inoperable chronic thromboembolic pulmonary hypertension. Heart 2013; 99: 1415-1420.

26 Chin KM, Kim NH, Rubin LJ. The right ventricle in pulmonary hypertension. Coron Artery Dis 2005; 16: 13-18.

27 van Wolferen SA, Marcus JT, Boonstra A, et al. Prognostic value of right ventricular mass, volume, and function in idiopathic pulmonary arterial hypertension. Eur Heart J 2007; 28: 1250-1257.

28 Reesink HJ, Marcus JT, Tulevski II, et al. Reverse right ventricular remodeling after pulmonary endarterectomy in patients with chronic thromboembolic pulmonary hypertension: utility of magnetic resonance imaging to demonstrate restoration of the right ventricle. J Thorac Cardiovasc Surg 2007; 133: 58-64.

29 Hardziyenka M, Campian ME, Reesink HJ, et al. Right ventricular failure following chronic pressure overload is associated with reduction in left ventricular mass: evidence for atrophic remodeling. J Am Coll Cardiol 2011; 57: 921-928.

30 Alpert JS. The effect of right ventricular dysfunction on left ventricular form and function. Chest 2001; 119: 1632-1633.

31 Li YD, Zhai ZG, Wu YF, et al. Improvement of right ventricular dysfunction after pulmonary endarterectomy in patients with chronic thromboembolic pulmonary hypertension: utility of echocardiography to demonstrate restoration of the right ventricle during 2-year follow-up. Thromb Res 2013; 131: e196-e201.

32 Delcroix M, Vonk Noordegraaf A, Fadel E, et al. Vascular and right ventricular remodelling in chronic thromboembolic pulmonary hypertension. Eur Respir J 2013; 41: 224-232. 